\title{
Use of a Rigid-Type Polyethylene T-Cannula, Silicone Tubing, and Adhesion Netting for Surgical Cannulation of Ileum of the Horse
}

\author{
Kohzo TANIGUCHI ${ }^{1)}$, Taketo OBITSU1), Hajime OHMURA ${ }^{2)}$, Atsushi HIRAGA ${ }^{2)}$, Hiroshi HATA ${ }^{3)}$, Hiroko AIDA ${ }^{2)}$, \\ Kentaro AKIYAMA ${ }^{2)}$ and Keiichi TANAKA ${ }^{4}$ \\ ${ }^{1)}$ Laboratory of Animal Nutrition and Feeding, Graduate School of Biosphere Sciences, Hiroshima University, Higashihiroshima 739- \\ $8528,{ }^{2)}$ Equine Science Division, Hidaka Training and Research Center, Japan Racing Association, Urakawa 057-0171, ${ }^{3)}$ Field Science \\ Center for Northern Biosphere and ${ }^{4}$ Laboratory of Animal Nutrition, Graduate School of Agriculture, Hokkaido University, Sapporo \\ 060-8589, Japan
}

(Received 20 December 2002/Accepted 14 April 2003)

ABSTRACT. A rigid-type of polyethylene T-cannula was fitted into the anterior ileum of six horses in order to improve the cannulation techniques. A piece of polyethylene net was fastened onto the intestinal wall around the cannula to prevent dislodgment of the cannula by promoting a secure adhesion between the ileum and the abdominal wall. The cannula barrel sheathed with silicone tubing was exteriorized through a stab incision at the lateral ventral wall on the transverse line of the second lumber vertebra, and a flange was screwed onto the barrel. The feeding regime gradually increased concentrate without roughage prevented any colic signs. The use of these techniques succeeded in the ileal cannulation with no leakage of digesta.

KEY WORDS: equine, ileum, polyethylene cannula.

J. Vet. Med. Sci. 65(8): 927-930, 2003

Most vertebrates obtain nutrients as products of digestion in the gastrointestinal tract, but the kind of nutrients is different among the digestion sites. Pre- and post-gastric fermentation provide short chain fatty acids and ammonia, and enzymatic hydrolysis in the small intestine glucose and amino acids to the animals. Additionally, the nutrient digestibility in the small intestine is different among feeds and animal species. Therefore, animal scientists have developed various cannulation techniques for each segment of the gastrointestinal tract to understand the digestion events. The efforts of research on the digestion of amino acids in the small intestine of pigs fitted with an ileal cannula led to the development of feeding standard for pigs on dietary amino acids. In ruminants, despite the widespread use of intestinal cannulas, there is no clear consensus on the best style of cannula, cannula material, or method of placement. These are especially critical factors, because the placement of cannula in the ileum causes many difficulties due to interference with the natural motility of the ileum and cecum [6].

Because horses have a larger cecum and colon and have a higher pressure of digesta flow in the ileum compared with ruminants, the ileal cannulation of horses is more difficult. Peloso et al. [9] fitted a silicone T-cannula into the ileum of ponies, and Coleman et al. [2] also used a similar flexibletype T-cannula constructed from plastisol (polyvinyl chloride). These flexible-type cannulas compared with rigidtype might have greater flexibility and less inflammation reaction by animal tissues, although the troubles of digesta leakage around the cannula barrel and colic were sometimes observed by researchers cited above. On the other hand, when the rigid-type T-cannula constructed from high-density polyvinyl chloride, all operated horses did not show signs of abdominal discomfort, although they had inflammation in the abdomen and the intestinal lumen around the cannula $[5,8]$. We adopted the high-density polyethylene T-cannula for the horse ileum with some modification.

The T-shaped cannula used for this study was constructed from high-density polyethylene (Fig. 1). The cannula size was similar to that of a small intestinal cannula used in cattle of similar body weight [11]. The cannula was fabricated by a manufacturer of polyethylene products (Sanko Plastic Co., Osaka) based on design specifications for the barrel, base, disk flange and cap. The cannula barrel was $16 \mathrm{~mm}$ inner diameter (i.d.), $24 \mathrm{~mm}$ outer diameter (o.d.) and $50 \mathrm{~mm}$ long. Polyethylene pipe $28 \mathrm{~mm}$ i.d., $35 \mathrm{~mm}$ o.d. and $60 \mathrm{~mm}$ long was used as the cannula base, which had a hole bored in the center before it was cut longitudinally. The barrel was inserted into the base hole, and welded to the base by heat fusion. The edge of the base was rounded and smoothed by

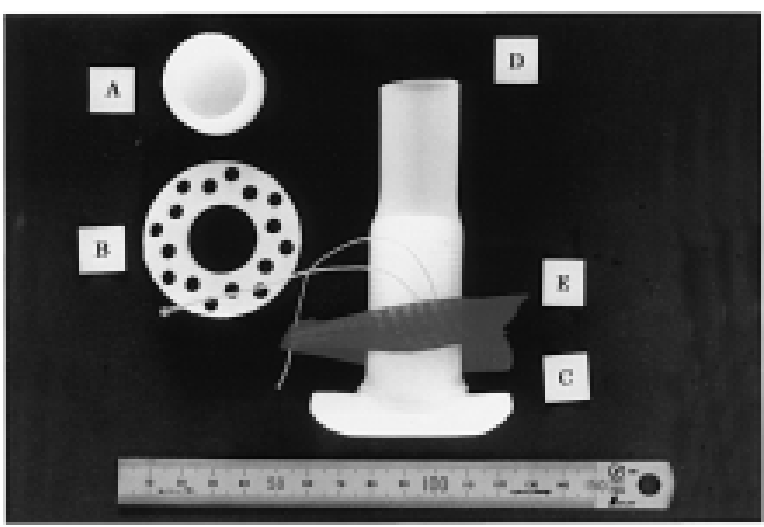

Fig. 1. The high-density T-cannula sheathed barrel with silicone tubing. A: cap, B: flange, C: barrel and base of cannula, D: silicone tubing, E: polyethylene net. 
a grinder to minimize intestinal irritation. A disk $50 \mathrm{~mm}$ in diameter and $5 \mathrm{~mm}$ thick was used for the cannula outside flange, which had 16 holes, $5 \mathrm{~mm}$, in addition to a center hole for the barrel. The screw thread of the barrel, flange and cap was $2 \mathrm{~mm}$. The inner side of the barrel edge was scratched in a $\mathrm{V}$-shape as a mark for the confirmation of cannula direction in the abdominal cavity after surgery. The cannula barrel was sheathed with silicone tubing, $19 \mathrm{~mm}$ i.d., $25 \mathrm{~mm}$ o.d. and $10 \mathrm{~cm}$ long, which was previously swollen by toluene. After then, the silicone tubing was recovered the original size by air-dry. The inner plug of the cannula barrel was prepared from hard styrene foam, which was cylindrical and $40 \mathrm{~mm}$ long. A wire was passed through the center of the styrene foam and fixed with rubber packing on both edges. A double-layer piece of elastic polyethylene net was used to promote adhesion of the cannulation site to the abdominal wall and prevent cannula dislodgment after surgery. The elastic polyethylene net that is normally used as fruit pouch at retail shops was pre-cut in pieces $70 \times 40$ $\mathrm{mm}$. A hole was cut in the center of the net to allow passage of the barrel when the net was expanded, and a polypropylene string was threaded around the center hole.

Surgical and animal care procedures for this research were in accordance with the guideline of the animal use regulation of Hiroshima University and also with the 'Guide for the Care and Use of Agricultural Animals in Agricultural Research and Teaching' [4]. Six Hokkaido native horses, geldings, with a mean body weight of $185.8 \pm 3.4 \mathrm{~kg}$ (standard error) and age of 14 months, were used. All horses were fed timothy hay and concentrate pellets, and vaccinated with tetanus toxoid prior to surgery. Feed was withheld $36 \mathrm{hr}$ and water $12 \mathrm{hr}$ before surgery. Pre-surgical preparation included clipping the ventral right flank, marking of the cannula position and placement of a 14-gauge, $13.3 \mathrm{~cm}$ catheter (Becton Dickinson, Tokyo) into the right jugular vein. The position marked on the abdomen of standing horses was $7 \mathrm{~cm}$ under the front of the right flank fold, and was $12 \mathrm{~cm}$ lateral to the ventral midline. The vertical distance from the paralumbar fossa, indicating the location of the cecal base and the ileocecal junction in the abdominal cavity, to the bottom of the abdomen averaged $45 \mathrm{~cm}$. Anesthesia was induced with ketamine $\mathrm{HCl}(2.5 \mathrm{mg} / \mathrm{kg})$ and guaifenesin (guaiacol glyceryl ether, $55 \mathrm{mg} / \mathrm{kg}$ ), following sedation with xylazine $\mathrm{HCl}(1 \mathrm{mg} / \mathrm{kg})$. General anesthesia was maintained with sevoflurane (2.8 to $3.3 \%$ ) delivered in oxygen via a large animal circle breathing circuit that includes the components for mechanical ventilation (LAVC-2000, JD Medical Distributing Com., Phoenix). Oxygen (100\%) flow rate was $3.0 \mathrm{l} / \mathrm{min}$. Lactated Ringer's solution was administered intravenously $(5 \mathrm{ml} / \mathrm{kg} / \mathrm{hr})$ using pre-indwelled catheter during anesthesia. The horse was placed in dorsal recumbancy on the surgery table with air mattress, the ventral abdomen was clipped, scrubbed and draped.

A celiotomy was performed on the ventral midline. The incision length of the skin including a layer of subcutaneous tissue was $20 \mathrm{~cm}$, beginning over the umbilicus and extended cranially. After hemorrhage from subcutaneous vessels was controlled, the linea alba was incised, and then the peritoneum was incised longitudinally along with the round ligament of the liver. The cecum apex was located immediately medial to the round ligament of the liver. When the dorsal band of the caecum body that led to the ileocecal junction was followed dorsally deep in the abdomen, the ileocecal fold and the distal ileum could be palpated.

The distal small intestine was exteriorized for the determination of the cannulation position, which was $30 \mathrm{~cm}$ cranial from the border of the ileocecal fold and about $60 \mathrm{~cm}$ cranial from the ileocecal junction. Although the gross distinction between the posterior jejunum and the beginning of the ileum was difficult to see during surgery, the middle of the anastomoses vessel of the last jejunum arteries with the cranial ileal arteries was used as a reference or landmark. The longitudinal length of the cecal body, from the ileocecal junction to the cecal apex, was averaged $52 \mathrm{~cm}$ in dorsal recumbecy. Thus, the cannula position could allow the movement of the cecum after surgery.

The minimal amount of intestine required for the cannulation was kept on moist towels, and the rest of the exteriorized intestine was put back in the abdominal cavity. The antimesenteric intestinal wall was excised in a slightly elliptical pattern for the cannula barrel, and incised longitudinally for the base. After the insertion of the cannula base into the intestinal lumen, the longitudinal incision was closed by Albert-Lembert suture pattern with suture size of 2-0, polyglactin 910, coated Vicryl (Ethicon, Johnson and Johnson Medical, Tokyo). A purse-string suture (2-0, polyglactin) around the cannula barrel was incorporated into the intestinal wall, and the cut edges of the intestine were inverted. A second purse string about $4 \mathrm{~mm}$ away from the first was placed snugly to complete the inversion of the secretory mucosa. A double-layer piece of elastic polyethylene net was put on the intestinal wall through the cannula barrel. The center hole of the net was ligatured with a polypropylene string around the cannula barrel, and the outskirts of the net was fastened at 8 points on the intestinal wall. To create a stoma, an electrocautery scalpel was used to remove the skin section marked on the abdominal wall; a circle of skin equal to the diameter of the cannula barrel was removed. A trocar of $2 \mathrm{~cm}$ diameter was exteriorized in the center of the circular stoma from the abdominal cavity through the peritoneum and muscle layers. A curved placental forceps was passed from outside into the stab wound made by the trocar to grasp the end of silicone tubing on the cannula, and to exteriorize the cannula barrel. After exteriorization and placement of the cannula in its final intended location, the part of silicone tubing visible outside the abdominal wall was cut off. After confirmation of no twist of the intestinal tract around the cannula in the abdominal cavity, a flange was screwed to fix the cannula in the abdominal wall. The ventral midline incision was closed in three layers. The peritoneum and the linea alba were closed together with a simple interrupted pattern of suture size 2 polyglactin including one vertical mattress suture as a ten- 
sion-relieving suture. The subcutaneous tissue was closed with a simple continuous layer of $2-0$ polyglactin. Skin staples were used for the closure of the skin. Peritoneal drainage was used for one horse, but not for the others because of the risk of the retrograde infection. The surgical procedures as mentioned above were carried out in five horses, but the procedures setting polyethylene net and silicone tubing were omitted in one horse.

All horses were administered xylazine $(0.25 \mathrm{mg} / \mathrm{kg}$, IV) during recovery from anesthesia. Flunixine meglumine (1.0 $\mathrm{mg} / \mathrm{kg}, \mathrm{IV}$ ) as analgesic was administered for 3 consecutive days and mixed antibiotics (IM) of procaine penicillin $(10,000 \mathrm{IU} / \mathrm{kg})$ and streptomycin $(13 \mathrm{mg} / \mathrm{kg})$ for 5 days following the surgery. Staples were removed at 12-14 days after surgery. The cannula parts out of the abdominal wall were cleaned with disinfectant solution every day for one month.

Horses were given two cubes of sugar and allowed to access to water and mineral blocks beginning $24 \mathrm{hr}$ after surgery. The first feeding of concentrate pellets, $100 \mathrm{~g}$ was at $48 \mathrm{hr}$ as moistened feed with water. The horses were fed 1 $\mathrm{kg} /$ day (d) of the moistened concentrate pellets from $\mathrm{d} 3$ to $\mathrm{d}$ 8 , and then gradually increased to $2 \mathrm{~kg} / \mathrm{d}$ as dried pellets until d 20. Eight cubes of sugar daily were added to the concentrate feed from d 3 to $\mathrm{d} 11$. Lucerne pellets, $400 \mathrm{~g} / \mathrm{d}$ was introduced to the concentrate pellets at $\mathrm{d} 9$, and gradually increased to $1.6 \mathrm{~kg} / \mathrm{d}$ at $\mathrm{d} 20$. A small amount of fresh grass was also given from d 13 to d 20. The daily meals was 5 times at $\mathrm{d} 3$ and 4 , and 4 times from $\mathrm{d} 5$ to $\mathrm{d} 20$. A diet consisting of concentrate pellets, lucerne pellets and chopped timothy hay was fed in three meals from $\mathrm{d} 21$. Bedding was not used in pens during 4 weeks, and the horses were handwalked once daily. As the result, the horses decreased their body weight by $20 \mathrm{~kg}$ from just before the surgery to $\mathrm{d} 9$ after surgery, and then increased to reach to the original body weight at $\mathrm{d} 21$. None of the horses showed signs of abdominal discomfort, such as colic, during the recovery period. In other surgical techniques [2,9], the horses sometimes demonstrated signs of colic within hours of feeding after consumption of grain and hay, when they were allowed to eat meals within $12 \mathrm{hr}$ after surgery. Early feeding of roughage after surgery of ileal cannulation in horse may impede or block flow of digesta, especially flow of fibrous contents, at the cannula base and cause leakage of digesta fluid and colic.

The five horses that received the cannula with silicone tubing did not have noticeable inflammation of the abdomen around the cannula, but the other one without silicone tubing had large inflammation. Although the inflammation spontaneously healed within two weeks, he not installed polyethylene net lost the cannula at 50 days after surgery, possibly due to catching on a stall gate or wall. The other five horses with no leakage of digesta were healthy at one month after surgery (Fig. 2), and were used for a digestion experiment. After then, they were killed for necropsy four months after surgery.

Post-mortem examination of horses prepared with poly-

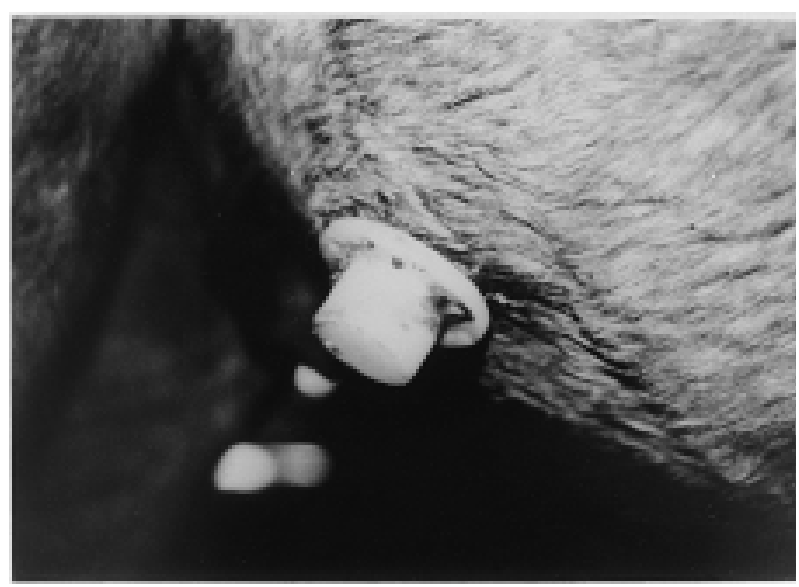

Fig. 2. The ileal cannula outside the abdominal wall at one month after surgery.

ethylene net showed that there was secure adhesion between the intestinal wall and the abdominal wall, eliminating the possibility of digesta escape into the abdominal cavity and the dislodgement of the cannula. The adhesion effects of polyethylene net have been reported on the cannulation for the rumen, abomasum and intestine of ruminants [11]. Other research workers used polyester fiber mesh [3] and polypropylene surgical mesh [10] with similar results in ruminants. Replacement of the porous sheet with an inner flange between the intestine and abdominal wall as reported in surgeries on ruminants might also result in tight permanent seal of a cannula $[1,11]$, but by using the porous sheet we avoided the potential complication of inflammation caused by irritation from the inner flange in our horses.

Gerhards et al. [5] and Leao et al. [8] installed the rigidtype cannula constructed from high-density polyvinyl chloride and succeeded in long term small intestinal fistulation of ponies, although they observed a potential build-up of intraluminal granulation tissue leading to the displacement of the cannula in a few case. Such intraluminal granulation was not seen in the present study. High-density polyethylene as cannula material compared with high-density polyvinyl chloride is characterized by the lighter weight, and works easily in cutting the cannula barrel for the adjustment of the length. The total weight of the cannula including the inner plug and the silicone tubing remaining after installation was only $42 \mathrm{~g}$. Aliyev [1] also succeeded in the long term use of high-density polyethylene as material for a reentrant cannula in cattle intestine.

Generally, use of rigid-type cannula material tends to promote opaque mucoid exudates around the cannula barrel in cattle [7, 10, 11] and horse [5], but other researchers [2, 9] used flexible-type cannula material did not describe such exudates in horses. The degree of stimulation of muscle tissue by the cannula barrel might affect exudation. That was one reason why we shield the cannula barrel with silicone tubing. However, this device could not completely suppress exudation, because granulation tissues of surface beneath 
the cannula flange were formed. The small holes of the cannula flange were useful for excretion of the exudates and for drying the tissues, but a zinc oxide ointment was also used for treatment of the tissues around the cannula. In sheep and goats, use of rigid-type cannulas was not accompanied by exudates and granulation tissues [11]. The nature and reaction of skin and muscles to the rigid-type cannula may be different among animals.

In conclusion, because the ileal cannulation of horse tends to cause the leakage of digesta, colics and dislodgment of the cannula due to the location on the abdomen, the materials for cannula construction is one of the important factors for successful maintenance and retention of the cannulas after surgery. The rigid-type cannula constructed from high-density polyethylene is lighter than cannulas made of other materials, and the use with polyethylene net helps to prevent the dislodgment of the cannula. Additionally, sheathing the cannula barrel with silicone tubing facilitates exteriorization during surgery and depresses unwanted tissue reaction after surgery. The techniques make it possible to carry out the studies on the nutrient digestion in the small intestine of horse, including the development of in vitro measurements as alternative methods.

ACKNOWLEDGEMENTS. The authors thank Yasuo Kobayashi, Dr. and Akira Matsui for their helpful co-operation. Matsuzaka Chiemi and Keiko Tokimura helped us for the managements of the operated horse. We are grateful to
Yo Asai, Dr. for the use of facilities of Hidaka Training and Research Center for this surgery. This work was supported by a Grant-in-Aid for Scientific Research (No. 12460116) from the Japanese Society for the Promotion of Science.

\section{REFERENCES}

1. Aliyev, A. A. 1982. Res. Vet. Sci. 32: 265-269.

2. Coleman, R. J., Mathison, G. W., Ingram, J. and Bell, D. 1998. Can. J. Anim. Sci. 78: 445-447.

3. Driedger, A., Condon, R. J., Nimrick, K. O. and Hatfield, E.E. 1970. J. Anim. Sci. 31: 772-775.

4. Federation of Animal Science Societies. 1999. Guide for the Care and Use of Agricultural Animals in Agricultural Research and Teaching. 1st rev. ed., Fed. Anim. Sci. Soc., Savoy.

5. Gerhards, H., Radiche, S. and Hipp, K. P. 1991. Pferdeheikunde 7: 243-248.

6. Harmon, D. L. and Richards, C. J. 1997. J. Anim. Sci. 75: $2248-2255$.

7. Komarek, R. J. 1981. J. Anim. Sci. 53: 796-802.

8. Leao, M. J., Almeida, F. Q., Carvalho, A. U., Valadares Filho, S. C., Oliveira, A. A. M. A. and Alvarenga, R. C. 1999. pp. 288-293. Proc. 16th Equine Nutr. Physiol. Symp., Raleigh, U.S.A.

9. Peloso, J. G., Schumacher, J., McClure, S. R., Crabill, M. R., Hanselka, D. V., Householder, D. D. and Potter, G. D. 1994. Can. J. Vet. Res. 58: 181-184.

10. Robinson, P. H., Smith, D. F. and Sniffen, C. J. 1985. J. Dairy Sci. 68: 986-995.

11. Taniguchi, K. and Obitsu, T. 1994. J. Fac. Appl. Biol. Sci. Hiroshima Univ. 33: 1-8. 